\title{
Effect of Calcium Supplementation on Glucocorticoid-Induced Osteoporosis
}

\author{
Hoda M Moghazy ${ }^{*}$, Aida Abdeen Mahmoud,**. \\ Rasha Abdeen Refaei,* , Mahmoud Raafat Abdel Fadeel, ***
}

* Department of Physiology, Faculty of Medicine, Sohag University, Sohag, Egypt.

**,Department of Biochemistry, Faculty of Medicine, Sohag University, Sohag, Egypt

***, Department of Physiology, Faculty of Medicine, Assiut University, Assiut, Egypt

\begin{abstract}
The commonest secondary cause of osteoporosis is glucocorticoid-induced osteoporosis (GIO) which is also the commonest cause of iatrogenic osteoporosis. Chronic glucocorticoids (GCs) treatment may cause fractures in $30-50 \%$ of patients. Fracture risk is increased specially among those using oral glucocorticoids. On longterm glucocorticoids therapy, deterioration of cortical bone progressively detected and long bones became increasingly fragile. Any patient with chronic glucocorticoid therapy should be suspected to suffer from GIO. Patients on glucocorticoids should receive supplementation with calcium. These deleterious influences are the result of suppression of bone formation that occurs with an early but temporal rise in the process of bone resorption. Numbers of osteoclast rapidly rise, and accompanied by inactivation of osteoblast with the decline of bone formation, these changes cause the fastest bone loss during the initial phase of the disease. Glucocorticoids cause inhibition of osteoblasts replication and function and accelerate their apoptosis. Calcium is fundamental for bone, and assuring sufficient calcium intake is important. It is said that when children and adolescents ingest large amount of calcium could result in a decrease in the danger of developing osteoporosis when they got old, however, some researches propose that solitary use of calcium supplementation might not be enough for lowering the danger for fractures. Conflicting results were reported about the effects of the use of supplemental calcium on the bone mineral content in those taking glucocorticoids therapy. Whereas, calcium supplementations were used in most bone protective treatment trials and therefore should be used as an adjunct to GIO therapy.
\end{abstract}

\section{Introduction}

Glucocorticoids (GCs) are vastly utilized for the therapy of different pathological conditions. However, chronic utilization of GCs possesses harmful effects on the skeleton, with a resultant reduction in the mass of bone and a rise in the hazard of fracture. It is reviewed that chronic glucocorticoids treatment may cause fractures in about $30-50 \%$ of patients. The commonest secondary cause of osteoporosis is Glucocorticoid-induced osteoporosis (GIO) [1].

GCs lead to a reduction of bone mass in 2 phases. First, a fast reduction in the mass of bone starts in the early 3 to 
6 months of GCs therapy, with a $6 \%$ to $12 \%$ decrease of bone mass in the first year of GCs therapy. Second, chronic utilization of GCs might lead to a $3 \%$ decrease in the mass of bone yearly. The hazard of fracture is already raised before a marked decline in the mass of bone happens. Therefore, it is very important to identify high-risk patients for the fracture to prevent these fractures resulting from GIO [2]. Nowadays, a fracture-risk assessment tool (FRAX) is used for fracture risk estimation [3].

Patients using prednisolone or any other glucocorticoid in a dose of $\geq 2.5$ mg daily for a period of $\geq 3$ months are advised to use sufficient calcium and vitamin D during GCs therapy. All patients on long-term GC therapy should have annual bone mineral density (BMD) testing, vertebral Xray, and fracture risk estimation utilizing FRAX [1].

Regarding the management strategies of osteoporosis, they include multiple aspects. General measures include smoking cessation, weight-bearing exercises, and ensuring a sufficient intake of calcium and vitamin D [4]. Osteoporosis therapy aims mainly to decrease the hazard of fracture. Each of calcium and medications is essential for achieving the mentioned goal. There is strong evidence supporting the beneficial effects of supplemental calcium on the skeleton and the hazard of fracture, especially when there is regular supplementation of calcium [5].

\section{Epidemiology of glucocorticoid- induced osteoporosis (GIO)}

Chronic oral glucocorticoid treatment is accompanied by rapid loss of bone and a rise in fracture risk which occurs within 3-6 months of therapy initiation and is also dose-dependent. The risk of fracture stays elevated during the duration of glucocorticoid treatment, but decrease after stoppage of treatment, although whether it returns to the baseline levels was unclear. Particularly vertebral fractures are characteristic of GIO, although the risk of other fractures, such as hip fractures, is also raised [6].

The effects of intravenous and inhaled glucocorticoids on the fracture risk are less well reported. Some researches reported that high doses of glucocorticoids taken by inhalation might be associated with a rise in fracture risk, although concurrent use of glucocorticoids orally is usually a confounding factor. Whereas, in a large case-control study, no rise in fracture risk was reported with the use of other forms of topical steroids [7].

\section{Pathophysiology glucocorticoid- induced osteoporosis (GIO) \\ Direct Effects of glucocorticoids on Bone}

Decreased bone formation is characteristic of GIO and is accompanied by an additional rise in bone resorption which occurs at an earlier time and is temporal [6].

Molecular mechanisms involved in the occurrence of GIO have been discussed in many studies, that include impaired differentiation of osteoblasts, increased apoptosis of osteocytes and osteoblasts, and prolonged lifespan of osteoclasts. Increased apoptosis of osteoblasts leads to a decline in the formation of bone, and it has been suggested that the apoptosis of osteocytes leads to an impaired response to bone damage and disruption of the osteocyte-canalicular network [8], that might lead to a reduction in bone strength. Additionally, osteoblasts apoptosis was discovered to be related to activation of glycogen synthase kinase $3 \beta$ (GSK $3 \beta$ ), that is involved in the Wnt signaling (Wingless-related integration site) pathway [9]. The Wnt signaling pathway has a 
significant role in the metabolism of bone and in the process of osteoblastogenesis. It has been recorded that GCs suppress the Wnt signaling pathway by enhancing the production of inhibitors of the Wnt pathway, such as sclerostin [10]. In this way, GCs induce suppression of differentiation of osteoblast. Additionally, glucocorticoids result in impairment of osteoblast function [11].

Also, GCs were found to induce bone marrow stromal cells, which are the precursor cells of osteoblasts, to differentiate into adipocytes instead of becoming osteoblasts. In contrast to increasing the apoptosis of both osteoblasts and osteocytes, GCs decrease the apoptosis of osteoclasts and prolong their lifespan [12].

Indirect Effects of glucocorticoids on Bone

GCs cause impairment of metabolism of bone through suppression of absorption of calcium through the digestive tract and also by suppression of reabsorption of calcium through the kidney, that might result in hypocalcemia and thus, later on, can lead to hyperparathyroidism [13]. Additionally, GCs also affect bone mineralization via transrepression of the matrix proteins, collagen I, and osteocalcin. Furthermore, GCs have deleterious effects on muscle mass and strength leading to muscle atrophy [14]. This GCs-induced muscle atrophy is associated with narrower bones with thinner bone cortices and reduced bending strength and impaired body balance, that in turn raises the risk for falls and bone fractures [12].

\section{Effect of calcium on bone health}

Calcium is referred to as the most plentiful mineral in human bodies. It is available in a certain food, as dietary supplements, and exists in certain drugs (including antacids). Below $1 \%$ of the calcium in the blood is required to attain important functions such as vasoconstriction and vasodilatation [15]. The rest of calcium in the body i.e. $99 \%$, is present in a stored form in our bone and also teeth and here calcium exists for supporting their construction and function. Bone is considered a dynamic tissue that undergoes continual remodeling, with persistent resorption and deposition of calcium in the newly formed matrix. Balance among both processes; bone resorption and deposition modifies according to age. The bone formation overrides bone resorption in the times of growth as in childhood period and adolescence, whereas in the periods of early and middle adulthood both processes occur approximately to the same level. While in older ages, particularly in postmenopausal females, bone resorption overrides bone formation, resulting in loss of bone which in turn increases the risk for osteoporosis with time [16].

Calcium is important for bone, and so guaranteeing enough calcium intake is essential. Adults aged over nineteen years old should take 1 gram $(\mathrm{gm}) /$ day. While females aged over fifty-one years, and all males aged $\geq 71$ years should take $1.2 \mathrm{gm}$ calcium daily. If the dietary intake is not adequate, calcium supplementation should be taken into consideration. Vitamin D plays an important role, as it is essential for the absorption of calcium from intestine. Dietary sources for calcium are saltwater fish, fortified foods, and liver. Whereas, the active form of vitamin $\mathrm{D}$, is not obtained from food but from sun exposure, so regular and moderate exposure to sunrays is advised [15].

\section{Role of calcium supplementation}

Great amounts of calcium intake in food and/or calcium supplements can markedly cause an improvement of bone mineral density in women in the postmenopausal period, while, certain 
researches suppose that the single use of supplemental calcium might not be enough to decrease the hazard of fracture and that co-supplementation with vitamin $\mathrm{D}$ is also required. Calcium, when supplemented with vitamin $\mathrm{D}$, decreases the hazard of fracture and rises the survival in elderly persons [17]. Additionally, ingestion of a great amount of calcium by the children and adolescents could result in a decrease in the hazard of osteoporosis in elderly persons and in postmenopausal women [18].

Conflicting results about the impacts of calcium on BMD in those taking GCs therapy have been reported. However, calcium supplementations were used in most regimens of bone protective treatment and therefore should be used as adjunct therapy [6].

The advised intake of calcium in patients taking glucocorticoids therapy is about 1-1.5 gm daily [19]. Regarding calcium preparations, calcium carbonate is the commonest used one. Whereas, calcium citrate is preferred in persons using the proton pump inhibitors and in those with achlorhydria. Regarding cholecalciferol, doses of 1000-2000 units daily are advised [20]. supplemental calcium has exhibited to impair the rate of loss of bone in patients taking glucocorticoids therapy although its effect on decreasing the risk of fracture was not reported [4].

Bolland et al. [21] performed metaanalyses of researches of different intake of calcium, involving estimation of fracture at the end of each study. They recorded that the advantages of supplemental calcium on the resistance to fracture are much fewer among older peoples. In another 26 researches, supplemental calcium, with or without vitamin $\mathrm{D}$, markedly decreased the hazard for whole body fractures and also vertebral fractures. Whereas, there are other 4 researches that exhibited no advantage of calcium supplementation on the fracture risk at any site in the body [21].

Also, another meta-analysis focusing on the impact of supplemental calcium on the BMD reported that supplemental calcium resulted in a little, early, and also non-advanced improvement in BMD that did not lead to a marked decrease in the risk for fracture [22].

Additionally, American peoples have among the highest intake of calcium worldwide, but also they have one of the highest incidences of osteoporosis. So, it is supposed that the role of calcium in increasing bone strength is still unclear [23]. Dairy products are the best source of calcium as they are not only the most bioavailable source but are also are high energy food source [24]. Dietary source for calcium is better than supplemental calcium, owing to the reduced risks of adverse effects, especially the cardiovascular effects and kidney stones. Patients should have knowledge about the potential side effects of supplemental calcium. Dietary calcium consumption, such as dairy products, however, does not seem to increase the danger for cardiovascular effects or kidney stones as seen in cases of using supplemental calcium [23].

Additionally, there is research reported that calcium supplements are ineffective when utilized as the primary defense against osteoporosis and fractures, and have been found to result in little improvement to BMD, even when calcium is supplemented with vitamin D [21].

\section{Conclusion}

We should be aware that using GCs treatment can result in side effects on bone. Therefore, caution with the use of GCs is recommended. The dose used should be at the minimum possible amount and be in the shortest 
duration of GCs treatment. Alternate day regimens are advised whenever prolonged therapy is planned, to maintain responsiveness of the hypothalamic-pituitary-adrenal axis. Long-acting and highly potent GCs (e.g. dexamethasone) are unsuitable for chronic GCs use. Additionally, protective measures should be adopted whenever possible to avoid the risk of GIO. During GCs therapy regular monitoring is needed to detect these adverse effects as soon as possible. Also it is concluded that calcium supplementation leads to a slight but not an apparent increase in bone mass in osteoporotic models supposing that calcium supplementation has only a minor role in the modern medical therapy of osteoporosis.

\section{References}

1. Park SY, Gong HS, Kim KM, Kim D, Kim HY, Jeon CH, et al. Korean Guideline for the Prevention and Treatment of Glucocorticoidinduced Osteoporosis. J Bone Metab. 2018; 25 (4): 195-211.

2. Seeman E, Delmas PD. Bone quality--the material and structural basis of bone strength and fragility. N Engl J Med. 2006; 354: 2250-2261.

3. Buckley L, Guyatt G, Fink HA, Cannon M, Grossman J, Hansen KE, et al. 2017 American college of rheumatology guideline for the prevention and treatment of glucocorticoid-induced osteoporosis. Arthritis Care Res (Hoboken). 2017; 69: 1095-1110.

4. Cherian KE, Kapoor N, Paul TV. Glucocorticoid-induced Osteoporosis. Indian J Endocrinol Metab. 2017; 21 (5): 652-654.

5.Porthouse J, Cockayne S, King C, Saxon L, Steele E, Aspray T, et al. Randomised controlled trial of calcium and supplementation with cholecalciferol (vitamin $\mathrm{D}_{3}$ ) for prevention of fractures in primary care. BMJ. 2005; 330 (7498): 1003.
6. Compston J. Glucocorticoid-induced osteoporosis: an update. Endocrine. 2018; 61 (1): 7-16.

7. Vestergaard P, Rejnmark L, Mosekilde L. Fracture risk associated with systemic and topical corticosteroids. J. Intern. Med. 2005; 257 (4): 374-384.

8. O'Brien CA, Jia D, Plotkin LI, Bellido T, Powers CC, Stewart SA, et al. Glucocorticoids act directly on osteoblasts and osteocytes to induce their apoptosis and reduce bone formation and strength. Endocrinology. 2004; 145 (4): 1835-1841.

9. Yun SI, Yoon HY, Jeong SY, Chung YS. Glucocorticoid induces apoptosis of osteoblast cells through the activation of glycogen synthase kinase 3beta. J Bone Miner Metab. 2009; 27 (2): 140-148.

10.] Wang FS, Ko JY, Yeh DW, Ke HC, Wu HL. Modulation of Dickkopf-1 attenuates glucocorticoid induction of osteoblast apoptosis, adipocytic differentiation, and bone mass loss. Endocrinology. 2008; 149 (4): 1793-801.

11. Butler JS, Queally JM, Devitt BM, Murray DW, Doran PP, O'Byrne JM. Silencing Dkk1 expression rescues dexamethasone-induced suppression of primary human osteoblast differentiation. BMC Musculoskelet Disord. 2010; 11: 210.

12.Raterman HG, Bultink IEM, Lems WF. Current Treatments and New Developments in the Management of Glucocorticoid-induced Osteoporosis. Drugs. 2019; 79 (10): 1065-1087.

13. Weinstein RS. Clinical practice. Glucocorticoid-induced bone disease. N Engl J Med. 2011; 365 (1): 62-70.

14. Canalis E, Mazziotti G, Giustina A, Bilezikian JP. Glucocorticoid-induced osteoporosis: pathophysiology and therapy. Osteoporos Int. 2007; 18 (10): 1319-1328.

15. Ukon $Y$, Makino $T$, Kodama $J$, Tsukazaki H, Tateiwa D, Yoshikawa H et al. Molecular-Based Treatment Strategies for Osteoporosis: A Literature Review. Int J Mol Sci. 2019; 20 (10): 2557. 
16. Ross A, Taylor C, Yaktine A, Valle H, Washington D. Institute of Medicine (US) Committee to review dietary reference intakes for calcium and vitamin D. USA: National Academies Press. 2011.

17. Saini AK, Dawe EJC, Thompson SM, Rosson JW. Vitamin D and Calcium Supplementation in Elderly Patients Suffering Fragility Fractures; The Road not Taken. Open Orthop. J. 2017; 11: 1230-1235.

18. Broe KE, Chen TC, Weinberg J, Bischoff-Ferrari HA, Holick MF, Kiel DP. A higher dose of vitamin d reduces the risk of falls in nursing home residents: A randomized, multiple-dose study. J Am Geriatr Soc. 2007; 55: 234-239.

19. Grossman JM, Gordon R, Ranganath VK, Deal C, Caplan L, Chen W, et al. American College of Rheumatology 2010 recommendations for the prevention and treatment of glucocorticoid-induced osteoporosis. Arthritis Care Res (Hoboken). 2010; 62: 1515-1526.
20.] Gupta N, Farooqui KJ, Batra CM, Marwaha RK, Mithal A. Effect of oral versus intramuscular Vitamin D replacement in apparently healthy adults with Vitamin D deficiency. Indian $\mathbf{J}$ Endocrinol Metab. 2017; 21: 131-136.

21. Bolland MJ, Leung W, Tai V, Bastin S, Gamble GD, Grey A. Calcium intake and risk of fracture: a systematic review. BMJ. 2015; 351: 4580.

22. Tai V, Leung W, Grey A, Reid IR, Bolland MJ. Calcium intake and bone mineral density: systematic review and meta-analysis. BMJ. 2015; 351: 4183.

23. Goolsby MA, Boniquit N. Bone Health in Athletes; The Role of Exercise, Nutrition, and Hormones. Sports Health. 2017; 9 (2): 108-117.

24. Lorincz C, Manske SL, Zernicke R. Bone health: part 1, nutrition. Sports Health. 2009; 1: 253-260 